\title{
Memory pattern identification for feedback tracking control in human- machine systems
}

\section{Miguel Martínez-García ${ }^{1}$ and Yu Zhang ${ }^{1}$ and Timothy Gordon ${ }^{2}$}

\section{abstract}

Objective: The aim of this paper was to identify the characteristics of memory patterns with respect to a visual input, perceived by the human operator during a manual control task, which consisted in following a moving target on a display with a cursor.

Background: Manual control tasks involve nondeclarative memory. The memory encodings of different motor skills have been referred to as procedural memories. The procedural memories have a pattern, which this paper sought to identify for the particular case of a onedimensional tracking task. Specifically, data recorded from human subjects controlling dynamical systems with different fractional order were investigated.

Method: A Finite Impulse Response (FIR) controller was fitted to the data, and pattern analysis was performed to the fitted parameters. Then, the FIR model was further reduced to a lower order controller; from the simplified model, the stability analysis of the human-machine system in closedloop was conducted.

Results: It is shown that the FIR model can be employed to identify and represent patterns in human procedural memories during manual control tasks. The obtained procedural memory pattern presents a time scale of about 650 ms before decay. Furthermore, the fitted controller is stable for systems with fractional order less or equal to 1 .

Conclusion: For systems of different fractional order, the proposed control scheme - based on a FIR model can effectively characterize the linear properties of manual control in humans.

Application: This research supports a biofidelic approach to human manual control modeling over feedback visual perceptions. Relevant applications of this research are: the development of shared-control systems, where a virtual human model assists the human during a control task, and human operator state monitoring.

- Keywords: human-machine interaction, information processing, memory, autonomous agents, adaptive

\footnotetext{
${ }^{1}$ Wolfson School of Mechanical, Electrical and Manufacturing Engineering, Loughborough University, UK

${ }^{2}$ School of Aeronautical, Automotive, Chemical and Materials Engineering, Loughborough University, UK

${ }^{3}$ School of Engineering, University of Lincoln, UK
}

\section{Corresponding author:}

Miguel Martínez García, Loughborough University, Wolfson School of Mechanical, Electrical and Manufacturing Engineering, Advanced VR Research Centre (AVRRC), Holywell Park, LE11 3TU, UK. Email: m.martinez-garcia@lboro.ac.uk 
automation, fractional order systems

\section{Introduction}

The execution of learned motor skills involves a series of coordinated actions by the muscular system. These actions are generally a response to perceived stimuli and are carried out automatically. Thus there is some type of nondeclarative memory involved in a manual control task. These memories, which encode motor skills, have been referred to as procedural memories (Squire, 2004). Examples of actions performed through procedural memories are: feedforward tasks, such as writing an English letter or language articulation (Kawato, 1999), and tasks which combine feedforward and feedback control, such as driving a vehicle or executing a pursuit control task (Donges, 1978; MartínezGarcía, Zhang, \& Gordon, 2016).

The aim of this paper was to identify procedural memory patterns, i.e. unconscious implicit memories that perform a complex activity, which are used by the human Central Nervous System (CNS) during manual control tasks with visual feedback. The term procedural memory is very broad and has been treated in different contexts in the literature. For example, cognitive architectures such as ACT-R represent procedural memories as a set of if-then rules, which contain the knowledge needed to achieve specific goals (Anderson \& Lebiere, 2014). These architectures can be employed to simulate human decision making processes and human behavior at a symbolic level. Thus they are tools that can aid in the design of human-machine interaction interfaces (Ritter, Baxter, Jones, \& Young, 2000). Recent versions of ACT-R include a vision module, which makes them suitable to model the motor responses to visual acquisition Ritter, Tehranchi, and Oury (2019). Other human cognition models are reviewed in Ritter (n.d.). Here, the particular case of procedural memory patterns in one-dimensional tracking tasks was studied. Hence, data collected from human subjects, executing compensatory and pursuit manual tracking tasks, were utilized in this paper.

A relevant motivation for this research was the design of shared-control systems, where a virtual human model assists the human in a control task - by simultaneously co-controlling a system. It is known that if the assisting virtual human model does not accurately characterize human control, this may lead to increased workload for the human operator, instead of improved performance (Griffiths \& Gillespie, 2005). The increased workload is more likely to occur when the model and the human produce control pulses in anti-phase, than when there is a relatively small magnitude discrepancy between the human and the model. Therefore, to achieve a higher degree of integration between the human and the machine, a better understanding of the principles of human control at a qualitative level is needed (Abbink et al., 2018; Wang, Zheng, Kaizuka, \& Nakano, 2018). One possible symbiotic control scheme is that of bi-manual control, in which a subject controls a machine with two controllers that combine their inputs; a real-world example is the control of an aircraft with a flying stick in one hand and the throttle in the other (Barfield \& Dazzo, 1983). With the subject of human-machine symbiosis in mind, the aim here was to produce a model that sketches the patterns of human control.

Many other different approaches are being investigated in academia to reproduce biofidelic manual control, particularly in the field of highway driving. These range from optimal control (Schmitt, Bieg, Manstetten, Herman, \& Stiefelhagen, 2016) and inverse optimal control (Inga, Eitel, Flad, \& Hohmann, 2018) to multiplicative models (Martínez-García \& Gordon, 2018).

The present study was performed under the assumption that the human operator response can be approximately characterized as a linear actuator. This is clearly an oversimplification, since the CNS is a complex network of paths, some wired in a feed-forward manner while others in a 
feedback manner, and therefore the human operator is a highly non-linear system (Bullier, 2001). Nevertheless, this approach is not uncommon in the literature; the theory of quasi-linear models (Jagacinski \& Flach, 2003; McRuer \& Jex, 1967; Tustin, 1947) shows that the human frequency response $H(s)$ can be represented as a superposition of a linear function $L(s)$ and some additional nonlinearities $R(s)$, which are usually called the remnant: $H(s)=L(s)+R(s)$. Although $L(s)$ cannot fully characterize human control, it still offers insights into the nature of the human responses. Additionally, for the control of simple plants (i.e., systems), the linear equivalent system $L(s)$ is sufficient to achieve stable control in closed-loop. In Roig (1962) it was estimated that for such systems $L(s)$ represents approximately $90 \%$ of the human control responses, although this number is highly variable and depends on the type of plant and control tasks being performed.

In this study $L(s)$ was fitted from the human data to a Finite Impulse Response (FIR) control system, which integrates a series of past observations through a weighted sum. In this way, the procedural memory pattern is here represented as a series of weights. These weights manifest the relative importance of the observations of a particular visual cue at different instants. Herein, the linear control responses of the human operator are modeled with a filter that considers the visual memories acquired during a control task - i.e., the dynamic displacements of a moving target.

The method of using a FIR model to mimic human control can be found in the literature (Shinners, 1974), but not the study of the resulting memory patterns, under the assumption that visual memories at different instants are weighted through an intrinsic procedural memory pattern.

Different biological mechanisms justify the existence of visual memories. For example, the CNS has an integration time of $\approx 100 \mathrm{~ms}$, which filters residual firing in the photoreceptors (Gregory, 1997). Furthermore, the human eye realizes approximately three saccades per second. During the brief saccadic periods visual short-term memory (VSTM) is used to remember the visual scene (Hollingworth, Richard, \& Luck, 2008). And, in Clifford and Ibbotson (2002) it was suggested that visual motion detection would be impossible without some sort of prefiltering of the perceived optical variables

Regarding the investigation of visual memories in manual control, a different approach was used in Martínez-García, Gordon, and Shu (2017), where the framework of fractional calculus was utilized. The fractional calculus approach offers the advantages of adding very few parameters to a model, and integrating well with general differential equations theory. However, because a fractional operator is specified with only one additional parameter, which represents the fractional order of differintegration, this method, although effective for modeling, has limited capacity to characterize procedural memory patterns. On the other hand, although the approach here employed (FIR model) requires a larger parameter set, it is more appropriate to study the characteristics of procedural memories in a control task. The investigated FIR model was here used as a human performance analysis tool.

\section{Method}

\section{Control Task}

When trying to characterize how the human operator processes a visual scene to elicit a control response, two caveats are present. First, when fitting a control model to the human response action, it is usually assumed that the parameters of the model are constant. In reality, human control is not a stationary process (Pauwelussen, 2012; Zhang, Martínez-García, \& Gordon, 2018). Secondly, it is difficult to identify which optical variables are extracted from the visual scene and integrated by the human CNS. Different optical variables have been proposed in the literature, from the displacements of the focus of expansion (Gibson, 1950) - produced by the perceived motion of the observer - to 
the flow lines radiating from the focus of expansion (Beall \& Loomis, 1996) - which can be perceived as a motion smear resulting from the filtering properties of the eye (Burr, 1980)*. To avoid these obstacles, simple laboratory tasks are commonly used in academia, where a moving target is presented in a display. Thus it is known what the human operator is responding to. Further, the dynamics of the target are driven by a stationary stochastic process. This is done with the intention of keeping the human control behavior approximately invariant, or equivalently within a fixed parameter range for the studied model (Pew, 2007). For these reasons, an equivalent methodology of a onedimensional tracking task was utilized in this research.

The experiments consisted of a dot following onedimensional tracking task. The experimental setup was composed of a $22^{\prime \prime}$ computer display and two control devices: a joystick (Logitech Extreme 3D Pro) and a steering wheel (Logitech G27). The joystick had a range of displacement of $\approx 15$ degrees in each direction, while the steering wheel could rotate 900 degrees lock to lock. The normalized displacement (from -1 left to 1 right) was recorded from the sensor of each device with a resolution of 12 bits, and amplified with a gain $K_{J}=2$ for the joystick and $K_{W}=5$ for the steering wheel. These gains were empirically tuned so that the tracking task had similar difficulty with both devices.

The display showed a graphical simulation representing the state of the controlled system and/or control error (Fig. 1), according to two different control modes: compensatory and pursuit (Martínez-García \& Gordon, 2016). In compensatory mode (Figs. 1a, Fig. 2a), the test subjects were required to nullify the error, which was the difference in position of a circular cursor - the follower from a fixed reference dot - or target - by using the control device (Fig. 1a). The dynamics of the controlled system were not explicitly displayed in compensatory mode; only the relative error was seen by the human. In pursuit mode
(Figs. 1b, 2b), the human had direct access to the plant dynamics represented by a moving follower dot. Herein, the forcing function acted by positioning the reference dot. In this case, the human subject perceived the error as the relative difference between the two moving dots (Fig. 1b), while using the control device as plant input. Usually, the human-control literature explores these two control modes (Jagacinski \& Flach, 2003). But in real applications, the distinction between them is not always clear. Nevertheless, as an example, ground vehicle driving is here considered; more or less, driving in a straight path in the presence of a lateral perturbation can be interpreted as a compensatory task, while following a curved path as a pursuit task.

* In highway driving, the flow lines can be easily assessed as the projected angles of the road boundaries on the retina: the splay angles (MartínezGarcía \& Gordon, 2018).

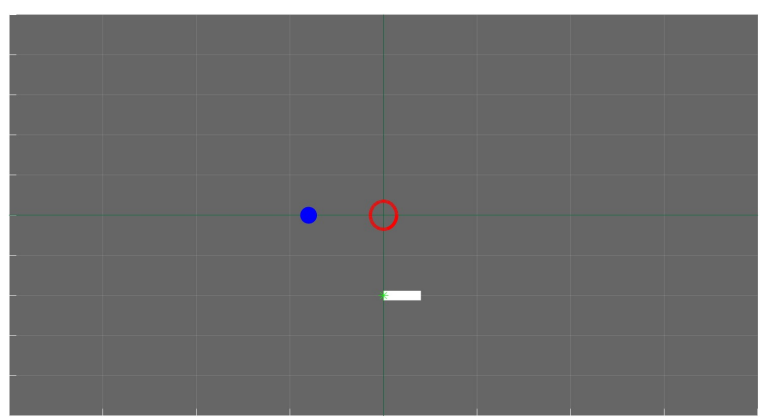

(a)

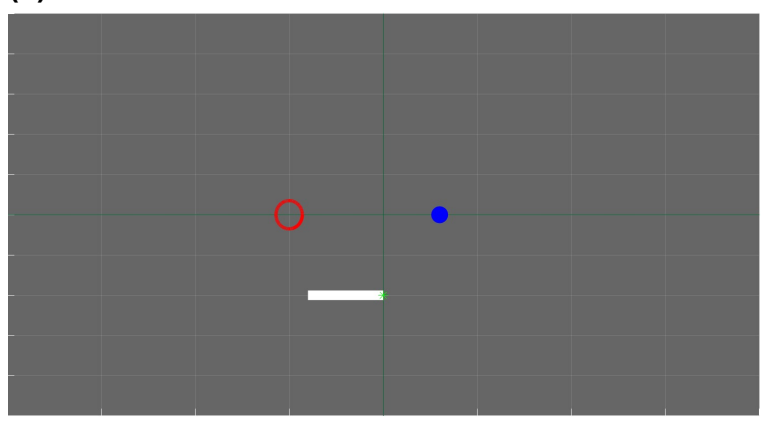

(b)

Figure 1. Display presented to the subjects during the experiments. The blue dot, controlled by the subject, follows the target - red circle. A white bar indicates the applied control gain. (a) In compensatory mode, the target is fixed at the center of the display, and the follower represents the difference between forcing function and controlled system output. (b) In pursuit mode, the target moves according to the forcing function while the follower marks the controlled system output. 


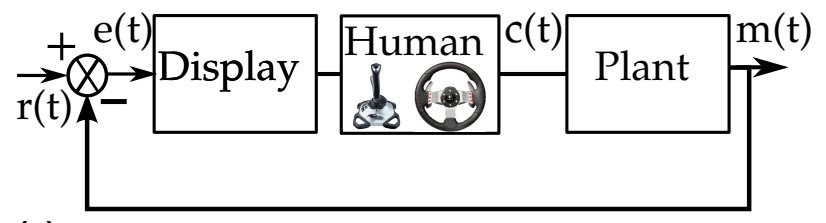

(a)

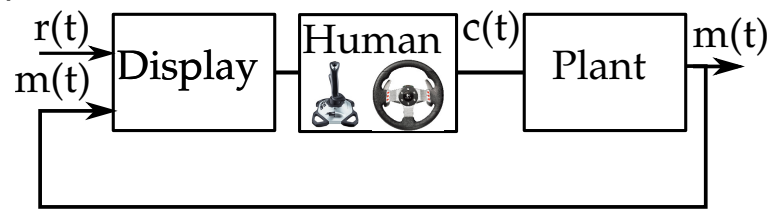

(b)

Figure 2. Human-machine control loop for the experimental setup in (a) compensatory mode and (b) pursuit mode.

The forcing function $r(t)$ was a sum of sinusoids with frequencies in the range $f_{k}=0.01-20 \mathrm{~Hz}$ :

$$
\begin{gathered}
r(t)=\sum_{f_{k} \in\{0.01,0.02, \ldots, 20\}} e^{-4 f_{k}} \sin \left(f_{k} \cdot 2 \pi t-\varphi_{k}\right) \\
\text {. }
\end{gathered}
$$

where $\varphi_{k} \in[-\pi, \pi]$ was a randomized phase for each term. With this choice, the amplitude was negligible for frequencies outside of the range where a human operator can perform adequate control - approximately up to $0.75 \mathrm{~Hz}$ (Wargo, 1967).

Each test was performed with five different controlled systems, described by the transfer function

$$
G_{\alpha}(s)=\frac{1}{s^{\alpha}(T s+1)},
$$

with $\alpha=0.5,0.75,1,1.25,1.5$ and $T=0.1$. The plants with $\alpha \neq 1$ are plants of fractional order. In the classical literature in human-machine systems (McRuer \& Jex, 1967), $G_{1}(s)$ is one of the commonly studied cases. Thus herein, variations of $G_{1}(s)$ are considered by incorporating negative $(\alpha<1)$ or positive $(\alpha>1)$ fractionality to the baseline system $G_{1}(s)$.

\section{Fractional Order Systems}

Plants with fractional order dynamics (2 with $\alpha \neq 1$ ) were employed to determine the capabilities of a human operator to control this type of dynamics. In Martínez-García et al. (2017) it was shown that humans have limited but consistent capability to compensate this category of plants. The reader may refer to this publication to retrieve more specific details about the experiments (briefly outlined in Table 1) and for a general overview of the mathematical aspects belonging to fractional calculus theory. For the investigation here conducted, it suffices to understand $\alpha$ as a parameter that allows to vary the order of a dynamical system in a smooth manner, instead of considering integer order transitions from one order of differentiation to the next. Smooth fractional variation of the exponents of a transfer function corresponds to smooth transitions in the slope of its magnitude in the frequency domain (Martínez-García et al., 2017). This permits for a more concise study on the effects of plant order variation on human control response. The fractional order operators are described through the following definitions of integral and derivative of non-integer (fractional) order (Podlubny, 1998):

Definition 1: Riemann-Liouville fractional integral. Given $\alpha \in \mathbb{R}^{+}$, a piecewise continuous function $f$ on the interval $(0, T]$, with $T \in \mathbb{R}^{+}$, and assuming that $f$ is integrable on the interval $[0, T]$, then for $t \in(0, T]$

$$
{ }_{0} D_{t}^{-\alpha} f(t)=\frac{1}{\Gamma(\alpha)} \int_{0}^{t} f(x)(t-x)^{\alpha-1} d x
$$

is the Riemann-Lioville fractional integral of order $\alpha$ at $t$.

\begin{tabular}{|l|c|}
\hline \multicolumn{2}{|c|}{ Experimental Parameters } \\
\hline \hline Number of subjects & 10 \\
\hline Age of subjects & $22-33$ years \\
\hline Duration of each event & $90 \mathrm{~s}$ (60 analyzed) \\
\hline Number of recorded events & 200 \\
\hline Forcing function freq. range & $0.01-20 \mathrm{~Hz}^{\star}$ \\
\hline Sampling frequency & $100 \mathrm{~Hz}$ \\
\hline Steering wheel range & -450 to 450 degrees \\
\hline \hline \multicolumn{2}{|c|}{ Optimization Parameters (system identification) } \\
\hline \hline Genetic algorithm \# generations & 1500 \\
\hline Genetic algorithm \# population & 500 \\
\hline
\end{tabular}

Table 1. Summarized experimental and optimization setup parameters. $(\star)$ The frequencies of the forcing function are spaced at $0.01 \mathrm{~Hz}$. Note that the frequencies are weighted so that they are only effective up to $0.5 \mathrm{~Hz}$. The remaining frequency values are added to simulate noise in the system. 
Definition 2: Riemann-Liouville fractional derivative. For $\nu>0$, the Riemann-Liouville fractional derivative of order $\nu$ is

$$
{ }_{0} D_{t}^{\nu} f(t)=\frac{d^{\lceil\nu\rceil}}{d t^{\lceil\nu\rceil}}\left\{{ }_{0} D_{t}^{\nu-\lceil\nu\rceil} f(t)\right\}
$$

where $\lceil x\rceil$ represents the ceiling function.

From (4) it is seen that fractional differentiation is defined from fractional integration; it involves computing first a fractional integral and then a classical derivative of order $\lceil\nu\rceil$. Thus fractional differentiation, unlike classical differentiation, is not a local operator, as it inherits the features of the integral operator.

Fractional order models are widely employed to represent systems with memory properties, such as viscoelastic materials and biological systems with complex interactions (Magin, 2006). However, the degree of fractionality is usually very small in real world systems; the transfer functions representing them, have exponents close to integer numbers. One example is a low fractionality model for ground vehicles (Martínez-García et al., 2017). In this paper, a larger degree of fractionality was introduced with exponents half a unit apart from the integer values (2). This was done to identify the adaptation characteristics of human control to plant order variation.

\section{Participants}

Ten human test subjects, with ages between 22 to 33 years and mixed genders ( 8 males, 2 females), took part in the human-control experiments voluntarily after signing a consent form $^{\dagger}$. Each subject performed 20 experimental trials lasting for 90 seconds each.

In total, 200 tracking experiments were recorded from the 10 participants. To minimize adaptation effects, half of the subjects performed the experiments with the joystick first, while this was reversed for the other half.

\section{Modeling Approach}

Humans employ a history of past observations - visual memories - to control a dynamical system. For instance, the particular wiring of retinal cells delays some visual inputs with respect to others to achieve temporal filtering properties (Kim et al., 2014). In Martínez-García et al. (2017) it was shown that the correlation between visual inputs and human control responses is increased when the visual inputs are considered to have fractional memory properties. Additionally, memory properties are ubiquitous in the neuromuscular system, from the oculomotor neurons (Anastasio, 1994) to the viscoelasticity of the arm muscles (Tejado, Valério, Pires, \& Martins, 2013). These past visual observations are integrated over specific time domain patterns or procedural memory patterns, which reflect the use of visual memories during manual control tasks. The hypothesis tested in this article was that these patterns can be, to some extent, characterized by a linear FIR model.

Herein, the linear component of human response was modeled with a FIR model, for the restricted case where the visual memories correspond to the one-dimensional dynamic displacements of a moving target (Fig. 1). The FIR model can be expressed as:

$$
l(t)=G_{H} \sum_{i=0}^{N} K_{i} \mathcal{L}^{[\rho+i]}\left\{e_{t}\right\}
$$

where $l(t)$ is the linear equivalent response, $G_{H}$ the human gain, $K_{i}$ the normalized visual cue weights (which show the decay of the visual cues over time), $e_{t}$ the tracking error at time $t$ and $\mathcal{L}$ the back-shift or lag operator. Thus in compensatory mode $e_{t}=e(t)$ (Fig. 2a) and in pursuit mode $e_{t}=r(t)-m(t)$ (Fig. 2b). The lag operator delays the error according to a constant term $\rho$ by $\rho \Delta T$ seconds, and a variable term $i$ by $i \Delta T$ seconds, where $\Delta T$ is the selected

\footnotetext{
$\dagger$ The experiments were approved by the College of Science Research Ethics Committee of the University of Lincoln with UID COSREC491.
} 
discretization step. The constant term $\rho$ reflects the reaction time of the human operator.

The human operator can be regarded as an intermittent controller acting at discrete time steps every $\Delta T=50 \mathrm{~ms}$. This assumption is justified by several research studies. In Craik (1947) it was pointed out that the human operator performs discrete ballistic corrections during a control task, although this may be difficult to detect in skilled operators (Craik, 1948), who rely more on memory and make smoother control responses (Miall, Weir, \& Stein, 1993). Also, it has been estimated that the time needed for the CNS to switch between different sensory channels is around $60 \mathrm{~ms}$. This value coincides with the minimum time gap between two signals that allows the brain to discriminate them (Wargo, 1967). In Card, Newell, and Moran (1983) it was reported that the cycle time of human perception - i.e. the time needed to decide how to respond to a stimulus - ranges from 25 to $170 \mathrm{~ms}$. This value is in agreement with the chosen $\Delta T$ and is relevant to estimate the optimal frame rate in a computer display (Chen \& Thropp, 2007). The two point discrete steering control model introduced in Salvucci and Gray (2004) also uses $\Delta T=50 \mathrm{~ms}$. Further, in Miall et al. (1993) it was suggested that intermittent control is a sign exhibited in negative feedback control; subjects deprived of visual feedback produce smoother control responses. Similarly, in Hollingworth et al. (2008) it was stated that human visual acquisition can be divided into a series of discrete steps. And in Martínez-García et al. (2016), fundamental discrete pulses were found from the steering signal in naturalistic driving data using signal decomposition methods, while in Zgonnikov and Markkula (2018) these pulses were modelled through an evidence accumulation mechanism.

The human response delay, here denoted by the parameter $\tau$, was included in the model to reflect the effective time delay with the additional transport delays during the control task. This value was determined in Zhang et al. (2018) for every subject and for the same data used in this study.
Accordingly, for this study, $\tau$ was pre-fixed with respect to the human subject's output for each controlled plant $G_{\alpha}(s)$ defined in Eq. 2, and averagely was $199 \mathrm{~ms}$ for joystick control and $272 \mathrm{~ms}$ for steering wheel control. From the value of $\tau$, the parameter $\rho$ was estimated for the FIR model from the relation $\rho \Delta T \approx \tau$.

\section{Model Parameter Fitting}

A genetic optimization algorithm was used to fit the weights $K_{i}$ in Eq. (5) to the human data. The objective function was the cross-correlation between $l(t)$ and the recorded human response - both controlling a plant acted by the same forcing function input. To compute $l(t)$ in the objective function, $\rho$ was fitted from the corresponding human subject data. During the optimization process, the gains $K_{i}$ were constrained to be a normalized vector, in order to prevent the generation of spurious patterns by the genetic algorithm.

The initial population of the algorithm was selected through a uniform probability distribution, while the parents in each generation were selected by the stochastic universal sampling technique (Baker, 1987). The mutations were produced by adding Gaussian noise to the sample and the crossover fraction in each generation was 0.8 .

Genetic algorithms are less prone to be captured at local minima, compared to other approaches such as convex optimization methods. Thus they have been consistently employed to fit parameters in generic data (Weiszer, Chen, \& Locatelli, 2015) - or when the objective function has unknown characteristics - such as data recorded from human subjects (Kase, Ritter, Bennett, Klein, \& Schoelles, 2017).

After the genetic algorithm was applied to determine $K_{i}$, the human gain $G_{H}$ was fitted by linear least squares. 


\section{Results}

\section{FIR Human Control Model}

To fit the weights of the FIR (5) to the human data, the genetic algorithm ran for a fixed number of generations and was executed multiple times with different randomizations (Table 1). For each run, the results were found to be very similar. The fitted parameters $K_{i}$ with respect to the number of delayed steps of length $\Delta T$ are shown in Fig. 3 for the 200 recorded experiments (20 per subject), including the joystick and the steering wheel.

Although there is large variability in the data (Fig. 3), a clear pattern is exhibited by the median values. Considering all the variants in the experiments - control device, type of plant, display mode and different subjects - and that the human operator response is in general very noisy, the variability was anticipated. Additionally, with the exception of $K_{3,4}$, the variability seems to increase with the number of delayed units (Fig. 3). For $i=2,3$ the high variability may be produced by the large slope of the trend. Thus the fact that it increases from $i=5$ while the magnitude of the weights decreases, confirms that humans rely in a more consistent manner on recent information than in older observations.

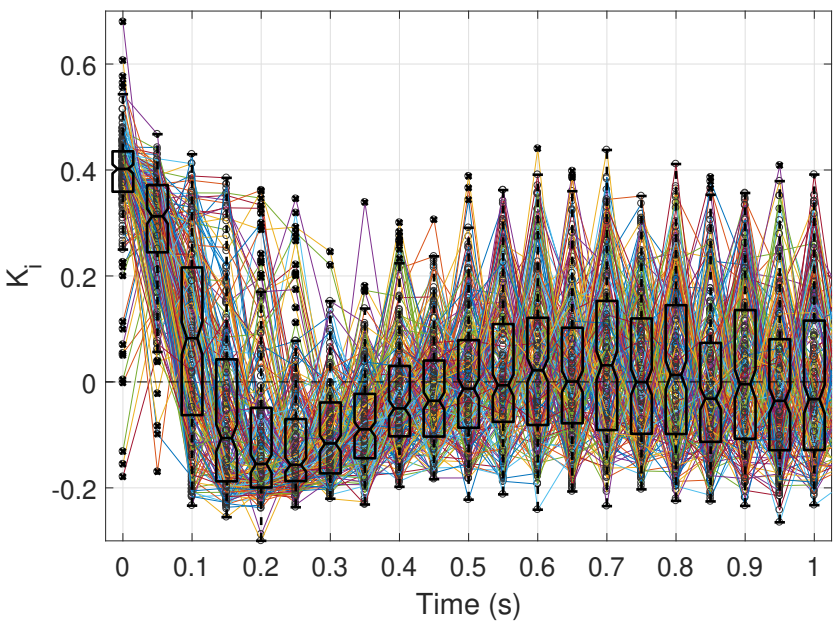

Figure 3. Fitted $K_{i}$ parameters with the experimental data recorded in this study. For each number of delayed steps of duration $\Delta T$, the corresponding box plot is displayed summarizing the 200 recorded experiments. In the box plots, the box edges mark the 25th and 75th percentiles while the box whiskers extend to 1.5 times the interquartile difference. The notches in the box plots indicate the range of the $95 \%$ confidence intervals for the median.
Fig. 3 also exhibits the resulting pattern of the human operator's adaptation to the plant $G_{\alpha}(s)$. It has been established (McRuer \& Jex, 1967) that the human response characteristics depend on those of the plant, in such a way that the whole human-machine system presents invariant dynamic properties. This is reflected by the evidence that the joint interaction of the human and the machine can be represented by a particular transfer function near the crossover frequency:

$$
C_{\omega_{c}}^{\tau}(s)=\frac{\omega_{c}}{s} e^{-\tau s}
$$

This transfer function (6) is known as the Crossover Model.

Here it was observed that, for the studied plants (2), the weights are positive for the first $2-3$ delayed steps (100$150 \mathrm{~ms}$ ) and remain negative during the continuing 8 delayed steps $(\approx 400 \mathrm{~ms})$. Finally, the weights become essentially zero after approximately 13 delayed steps $(650 \mathrm{~ms})$. This time constant shows the intrinsic time scale of the procedural memory pattern and is in agreement with Card et al. (1983), where it was reported that the decay time of a visually perceived image in memory is in the range 90$1000 \mathrm{~ms}$. For the chosen plant dynamics, control device and forcing function, it seems that humans use visual memory approximately up to $650 \mathrm{~ms}$. The sign change in the memory pattern between $K_{2}$ and $K_{3}$ manifests that, visual inputs perceived at different time are weighted in such a way that the rate of change of the error - and higher order rates - can be approximated by the human, allowing the CNS to estimate the future states of the observed system (7). Another possibility is that the shift in sign is associated at a physiological level with event-related potentials, such as contingent patterns of response in the frontal cortex (Walter, 1964).

Thus the FIR model is consistent with the concept that the human brain uses composite variables, which are linear combinations of derivatives of different orders of 
the observed cues, to predict the states of the tracked system (Hanneton, Berthoz, Droulez, \& Slotine, 1997). By considering that derivatives can be approximated as finite-differences it can be interpreted that, modeling the human operator with a FIR transfer function is qualitatively equivalent to considering the prediction effects of higher order derivatives. That is, as the $n$-th order derivative of a function $f$ can be approximated with

$$
D^{n} f(t) \approx \frac{1}{\Delta_{t}^{n}}\left\{\sum_{k=1}^{n}(-1)^{k}\left(\begin{array}{l}
n \\
k
\end{array}\right) f\left(t-k \Delta_{t}\right)\right\},
$$

which alternates positive and negative weights over past observations for the integer order case; humans may use the weight sign shift to obtain information about the rate of change of the controlled system - of integer or fractional order - by using visual memories. For example, in Hanneton et al. (1997) it was studied the case of human's using a linear combination of position and rate information $-a$ sliding variable - to predict the states of the controlled system.

\section{Pattern Analysis Across Experimental Variants}

The pattern observed in the weights $K_{i}$ (Fig. 3 ) is consistent across different subjects, after compensating their intrinsic effective time delay (Fig. 4a). Thus the number of test subjects in the experiments was sufficient. The pattern is also qualitatively consistent across different control devices (Fig. 4b) and within the different control modes (Fig. 4c). Hence, the selected model (5) and the optimization methodology are adequate, and the data have not been overfitted.

Nevertheless, in relation to the two types of control device tested, the observed pattern has quantitative differences between the joystick and the steering wheel. In Fig. 4b it is shown that for the case of joystick control, there is a higher negative gain but a faster weight decay, while for steering wheel control humans tend to use lower cue weights in magnitude but sustain the response longer. This effect is likely to be produced by the longer transport delays, which result from muscle latency, when using the steering wheel (Martínez-García \& Gordon, 2016).

Contrarily, the pattern is virtually identical between compensatory and pursuit mode (Fig. 4c). In another study with the same data (Martínez-García \& Gordon, 2016), it was shown that humans display a larger crossover frequency and greater performance in pursuit tasks than in compensatory tasks. Thus every aspect of the human response can be represented by the proposed methodology, but the approach is particularly useful to characterize visual memory patterns.

In Fig. $4 d$ the results are compared for the studied plants (2). It is observed that as the order of the plant increases, the magnitude of the gains decreases for recent observations, while the decay of the weights (5) over the perceived error is lower. This is possibly caused by the fact that the response of higher order plants is more difficult to predict, thus humans rely more on recent observations when the order of the plant is larger.

Further, the gains, $G_{H}$ in (5) are shown in Fig. 5 tabulated per subject. The gains are larger for joystick control than for steering wheel control, which is natural since the joystick has a higher output/hand-displacement ratio. Another reason is that untrained humans are not so skilled in joystick control and their output is closer to a bang-bang controller as compared to steering control; in steering wheel control both hands can act in anti-phase, increasing the impedance of the steering action to reduce instabilities (Burdet, Osu, Franklin, Milner, \& Kawato, 2001).

It was also observed that the gains increase with the order of the plant. As already stated, in general higher order plants are more difficult to control and usually require longer preview time. In Ito and Ito (1975) the effects of different preview time intervals were thoroughly examined. For the presented analysis, and in order to simplify the discussion, a preview of the error was not considered. 
(a)

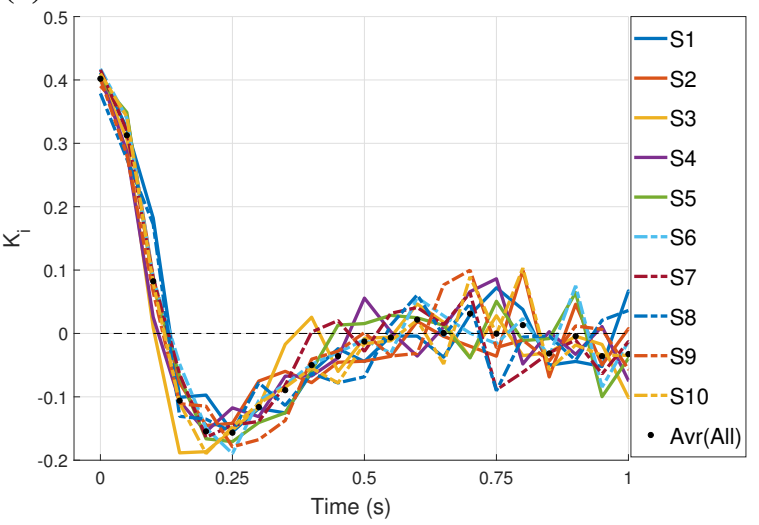

(b)

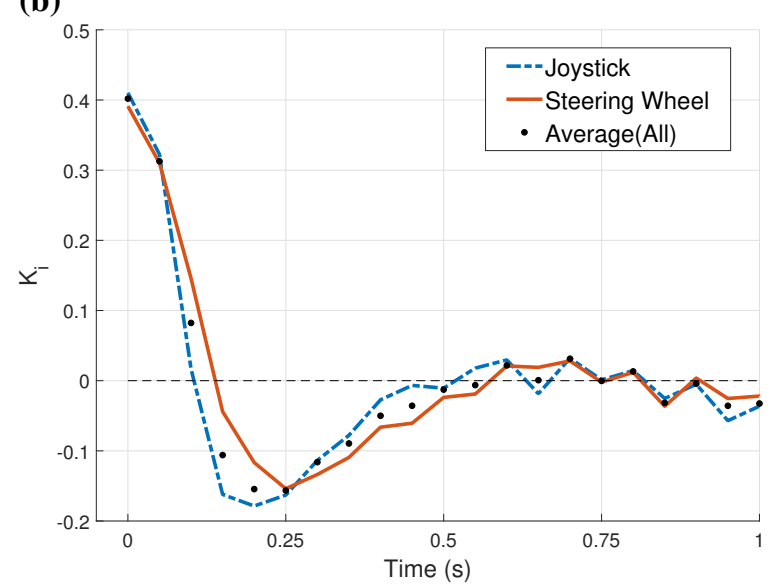

(c)

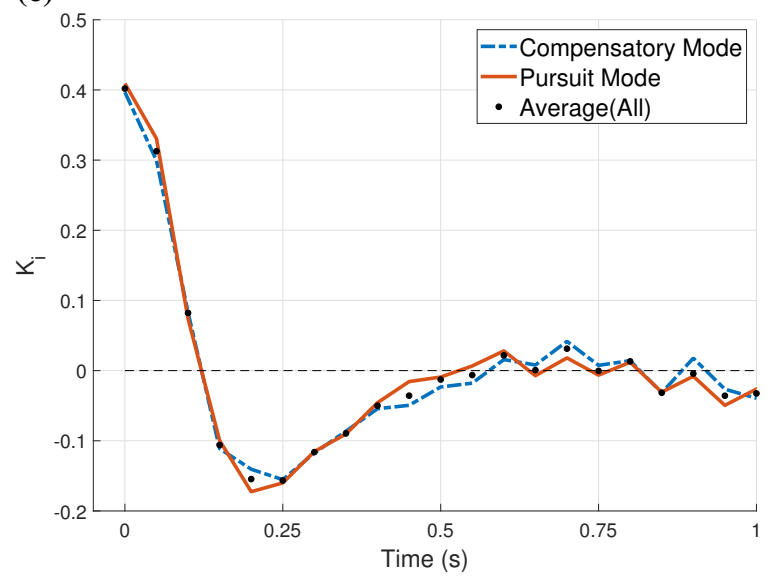

(d)

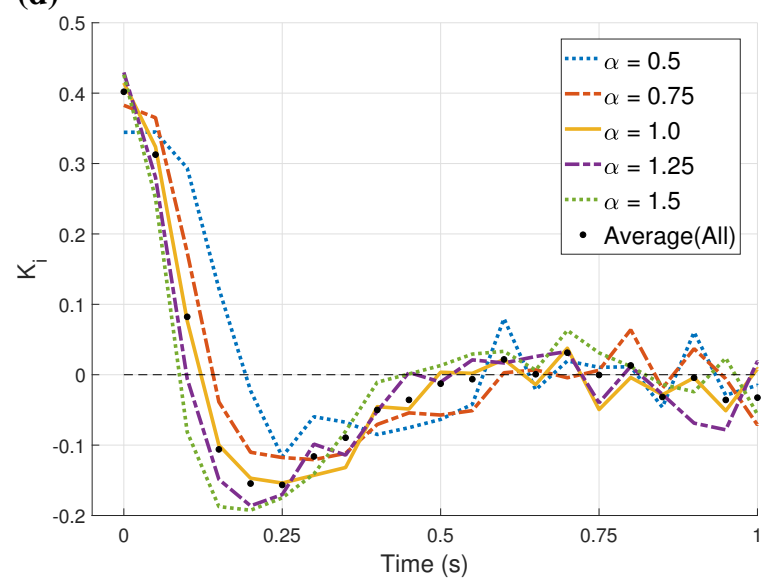

Figure 4. Fitted parameters $K_{i}$ averaged (a) for each subject (b) for different control device - joystick and steering wheel - (c) for different control modes - compensatory and pursuit - and (d) for each plant $G_{\alpha}(s)$.

\section{Reduced Order Model}

To study the FIR human control model from the perspective of classical control, it is practical to reduce it to simpler transfer functions. First, the FIR model was truncated by discarding the noisy cue weights in (5) - only $K_{0}$ to $K_{13}$ were considered:

$$
\tilde{H}(z)=G_{H}\left\{K_{0} z^{-\rho}+K_{1} z^{-\rho-1}+\cdots+K_{13} z^{-\rho-13}\right\} .
$$

Then, a lower order model - with two zeros and two poles was fitted from (8):

$$
H_{\alpha}^{d}(z)=\frac{a_{0}(\alpha)+a_{1}(\alpha) z^{-1}+a_{2}(\alpha) z^{-2}}{b_{0}(\alpha)+b_{1}(\alpha) z^{-1}+b_{2}(\alpha) z^{-2}}
$$

(a)

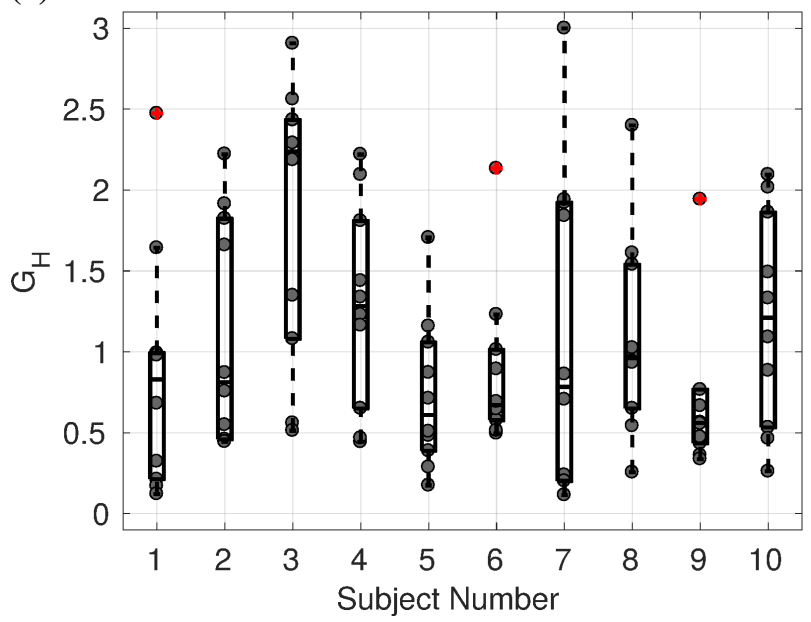

(b)

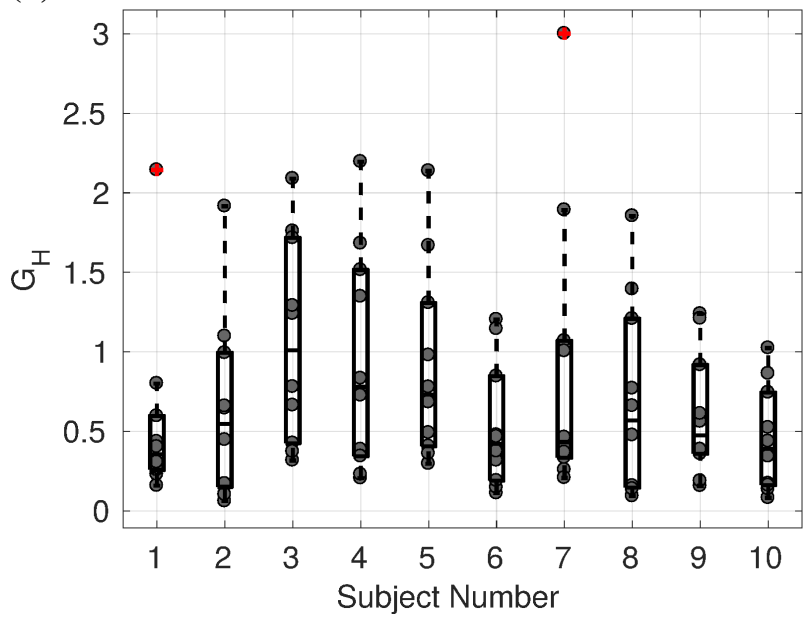

Figure 5. Fitted gain $G_{H}$ for the different human subjects for (a) joystick control and (b) steering wheel control. In the box plots, the box edges mark the 25th and 75th percentiles while the box whiskers extend to 1.5 times the interquartile difference. 


\begin{tabular}{|l||c|c|}
\hline Plant $\alpha$ & $H_{\alpha}^{d}(z)$ (Discr.) & Cont. $H_{\alpha}^{c}(s)$ (Cont.) \\
\hline 0.5 & $\frac{0.37-0.15 z^{-1}-0.11 z^{-2}}{1-1.47 z^{-1}+0.63 z^{-2}}$ & $\frac{0.13 s^{2}+12.32 s+57.15}{s^{2}+9.546 s+83.86}$ \\
\hline 0.75 & $\frac{0.39-0.19 z^{-1}-0.17 z^{-2}}{1-1.4 z^{-1}+0.56 z^{-2}}$ & $\frac{0.13 s^{2}+14.96 s+14.51}{s^{2}+11.72 s+68.07}$ \\
\hline 1.0 & $\frac{0.42-0.25 z^{-1}-0.16 z^{-2}}{1-1.37 z^{-1}+0.53 z^{-2}}$ & $\frac{0.18 s^{2}+16.09 s+8.08}{s^{2}+12.92 s+91.47}$ \\
\hline 1.25 & $\frac{0.45-0.31 z^{-1}-0.14 z^{-2}}{1-1.29 z^{-1}+0.48 z^{-2}}$ & $\frac{0.22 s^{2}+17.14 s-2.96}{s^{2}+14.97 s+111.2}$ \\
\hline 1.5 & $\frac{0.42-0.27 z^{-1}-0.17 z^{-2}}{1-1.25 z^{-1}+0.48 z^{-2}}$ & $\frac{0.19 s^{2}+17.20 s-16.27}{s^{2}+15.31 s+132}$ \\
\hline
\end{tabular}

Table 2. Lower order discrete transfer functions $H_{\alpha}^{d}(z)$ fitted from the FIR model (8) for each of the studied controlled plants $G_{\alpha}(s)$, and their continuous correlate $H_{\alpha}^{c}(s)$ obtained through the Tustin's bilinear transform.

The optimal lower order model $H_{\alpha}^{d}(z)$ was found through the instrument variable method from (8). The fitted models are shown in Table 2 and their corresponding frequency response in Fig. 8. The frequency response for the reduced model in (9) is accurate within the frequency range where the human operator can perform a fair level of control; the maximum frequency limit is in the range $1-5 \mathrm{~Hz}$, depending on the predictive capacity of a given manual control task (Pew, 2007). Another study (Wargo, 1967) stipulates a limit of $0.7-1.5 \mathrm{~Hz}$ for compensatory and pursuit tasks with irregular forcing functions, which do not allow for adequate prediction.

For the truncated FIR model (8), the pulse and step response are shown in Fig. 6. These can be compared to the pulse and step response of the reduced lower order FIR model (9) in Fig. 7. The lower order model, is a smoother variant of the FIR model. In both models the step response has a bounded steady state. However, for $\alpha>1$ the step response stabilizes at a negative value. This suggests that the controller may be unstable for such plants. Nonetheless, the pulse response of $H_{1}^{d}(z)$, representing the average control behaviour for all $\alpha$, is within the $95 \%$ confidence intervals established in Fig. 3, up to the limit of the here inferred time constant of $650 \mathrm{~ms}$ (Fig. 7). Thus the reduced model still matches faithfully the data.

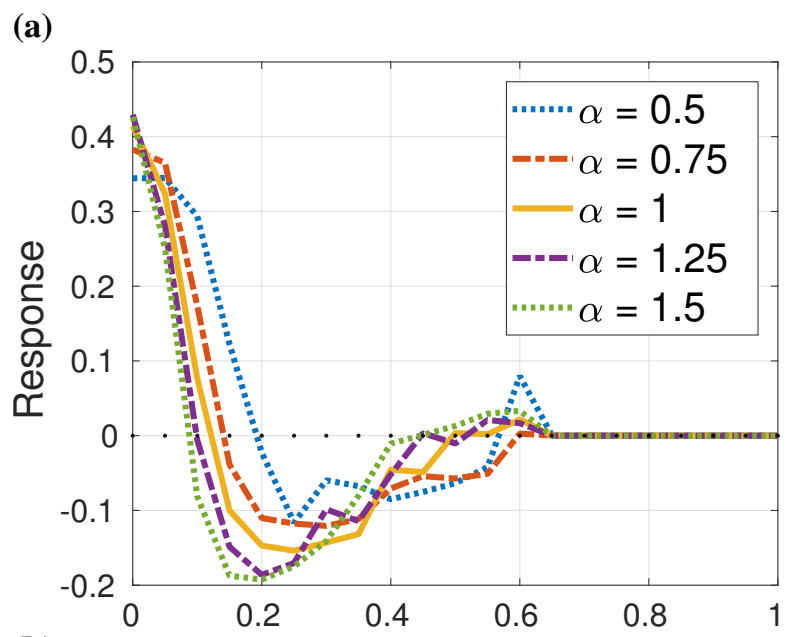

(b)

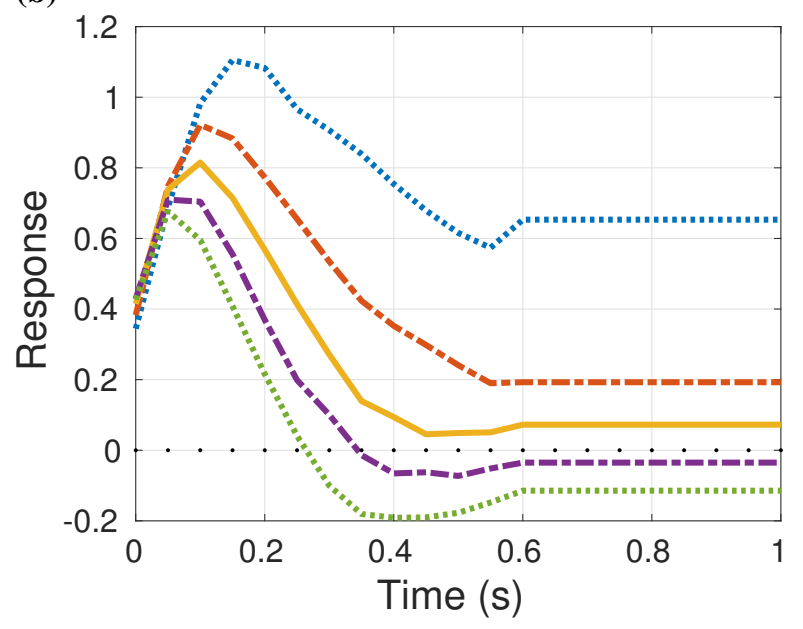

Figure 6. (a) Pulse response and (b) step response for the truncated FIR model in (8), controlling $G_{\alpha}(s)$ for each fractional coefficient $\alpha$.

\section{Stability Analysis}

Next, to analyze the stability of the reduced model (9) in closed-loop, the discrete approximation of the controller $H_{\alpha}^{d}(z)$ was converted to an infinite impulse response (IIR) controller $H_{\alpha}^{c}(s)$ (Table 2):

$$
H_{\alpha}^{c}(s)=\frac{c_{0}(\alpha) s^{2}+c_{1}(\alpha) s+c_{2}(\alpha)}{d_{0}(\alpha) s^{2}+d_{1}(\alpha) s+d_{2}(\alpha)} .
$$

Although as mentioned, the human operator can be regarded as a discrete controller, the filtering capabilities of the muscular system make the human motor response similar to a continuous signal (Craik, 1947). In classical control theory, a common way to analyze the stability of a system with feedback - for varying values of a parameter representing the control gain - is the so-called root locus 


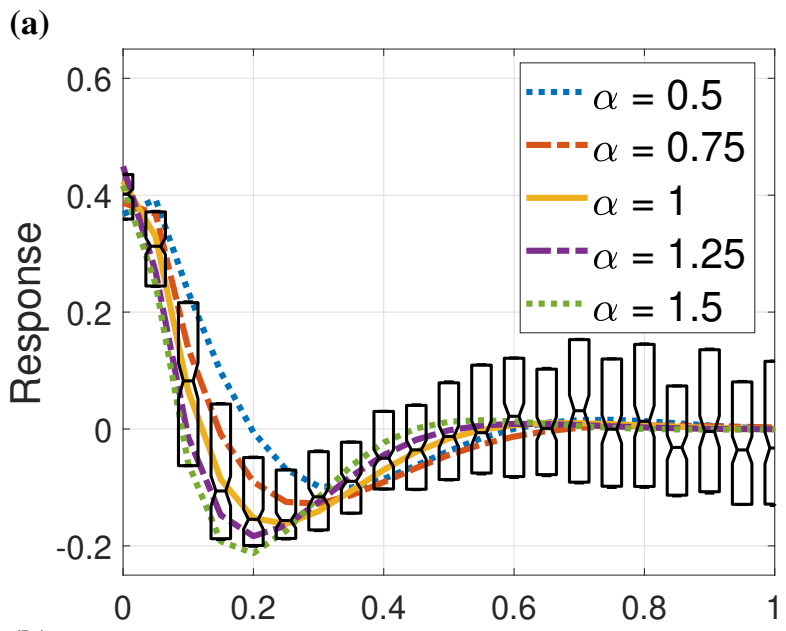

(b)

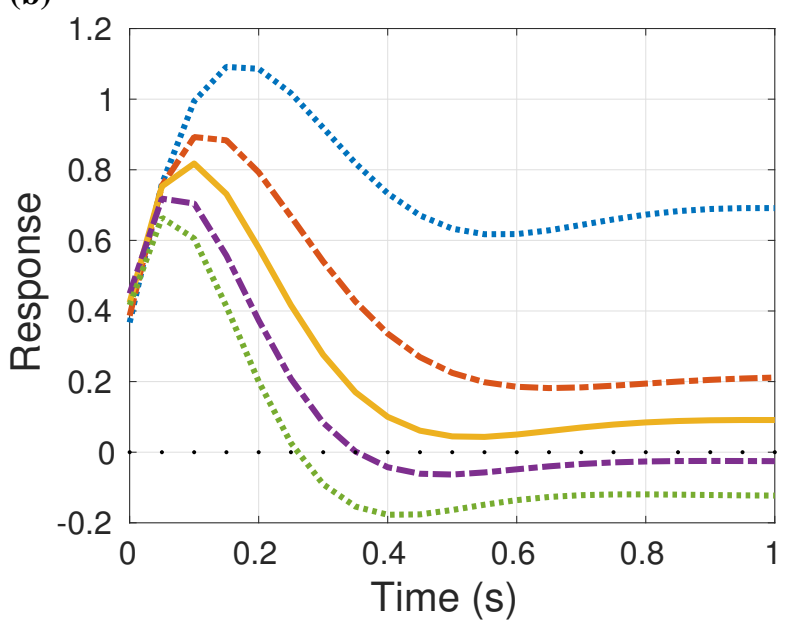

Figure 7. (a) Pulse response and (b) step response for the reduced lower order model $H_{\alpha}^{d}(z)$ (Table 2) controlling $G_{\alpha}(s)$ for each $\alpha . \ln (\mathrm{a})$, the notches in the box plots indicate the $95 \%$ confidence intervals for the median found in Fig. 3.

analysis. This method tracks the position of the poles for the different values of a parameter, which here is the control gain of the human operator in response to the visual memories of the input error.

In a root locus analysis, each pole of the closed-loop transfer function is represented as a path in a plot, where each point of the path marks the position of the pole for a particular gain. For the system to be stable with a particular gain, all the poles must be in the left half-plane.

To perform root locus stability analysis in the context studied here, there was one difficulty: the classical root locus technique is not applicable for fractional order transfer functions. The reason is that fractional order systems have branch points instead of poles. However,
1998). This theorem translates the bounded-input, boundedoutput (BIBO) stability region of a fractional order transfer function to the stability region of a non-fractional one.

Theorem (of stability for fractional order systems).

Given a fractional order transfer function $G(s)$ and a nonfractional transfer function $\tilde{G}(s)$ such that, for a particular $0<\rho<1$, they satisfy $\tilde{G}\left(s^{\rho}\right)=G(s)$ then,

$$
G(s) \text { is BIBO stable } \Longleftrightarrow|\arg (p)|>\rho \frac{\pi}{2}
$$

for every pole $p$ of $\tilde{G}(s)$.

In particular for $\rho=1$ the theorem coincides with the classical stability criteria for non-fractional transfer functions. Choosing $\rho=1 / 4$ as a common factor, all the plants $G_{\alpha}(s)$ were transformed into non-fractional order plants $\tilde{G}_{\alpha}(s)$ :

$$
\tilde{G}_{\alpha}(s)=\frac{1}{s^{4 \alpha}\left(T s^{4}+1\right)}
$$

And applying the same transformation $\left(s \rightarrow s^{4}\right)$ to a Padé approximation of the human response delay, and to the human control model $H_{\alpha}^{c}$ (Table 2), allowed for the computation of the root locus plot of the combined humanmachine system (Fig. 9). Each sub-figure shows the branches traced by the poles of the closed-loop system as the gain of the control loop is increased from 0 (start of the branch denoted by $\times$ ) to $\infty$ (end of the branch denoted by $\circ$.). Thus when a particular path is fully inside the gray - unstable region, the system is unstable for any chosen gain. Figs. 9a, $9 \mathrm{~b}$ and $9 \mathrm{c}$ show that the system is stable for $\alpha \leq 1$, as for these plants sufficiently small enough gains keep all the poles within the stability region (white background region). On the other hand, Figs. 9d and 9e show that the system cannot attain stability for any positive gain when $\alpha>1$. For $\alpha>1$ one of the branches starting at the origin is fully inside the unstable region (gray colored region) for positive gains. 
(a)

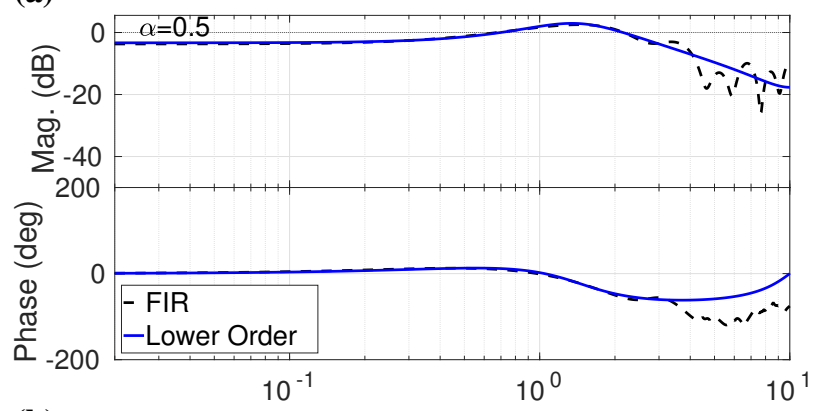

(b)

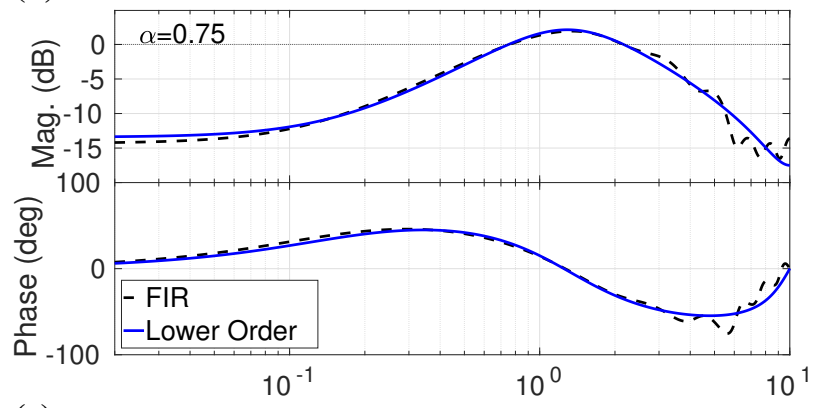

(c)

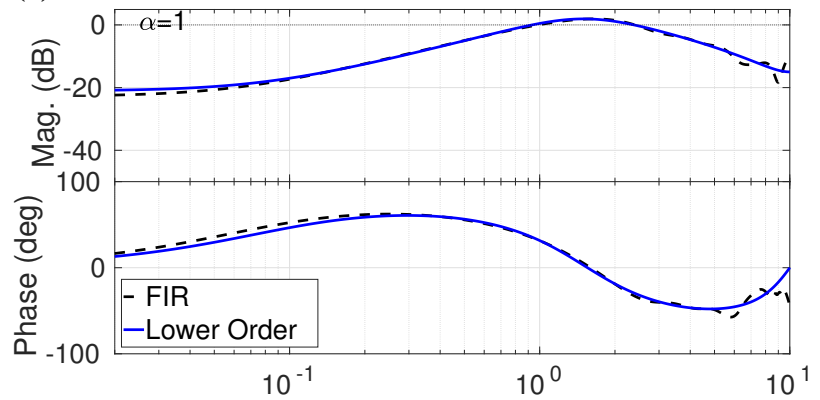

(d)

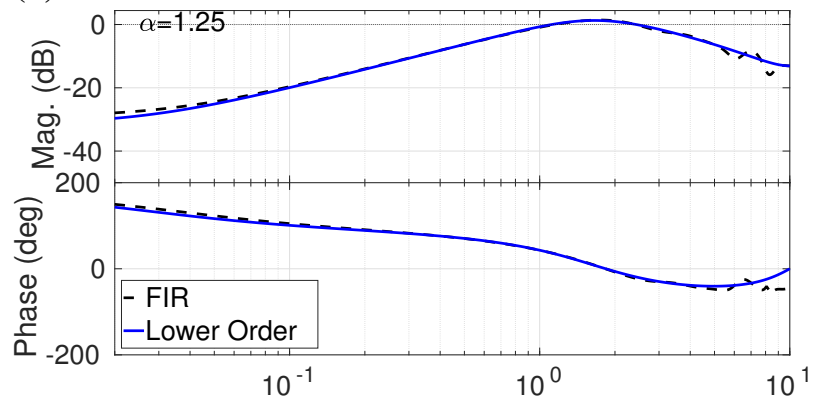

(e)

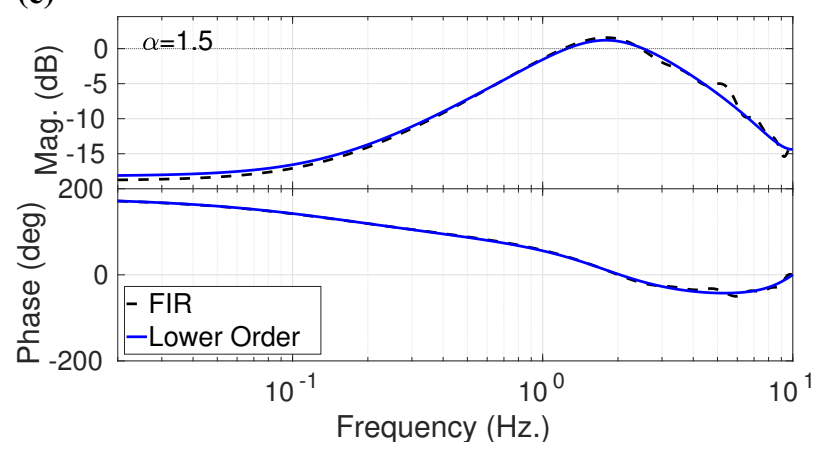

Figure 8. Frequency response for the FIR model (8) and its corresponding reduced order counterpart (9) for controlling the plants $G_{\alpha}$ (2) with (a) $\alpha=0.5$, (b) $\alpha=0.75$, (c) $\alpha=1.0$, (d) $\alpha=1.25$ and $(\mathrm{e}) \alpha=1.5$. (a)

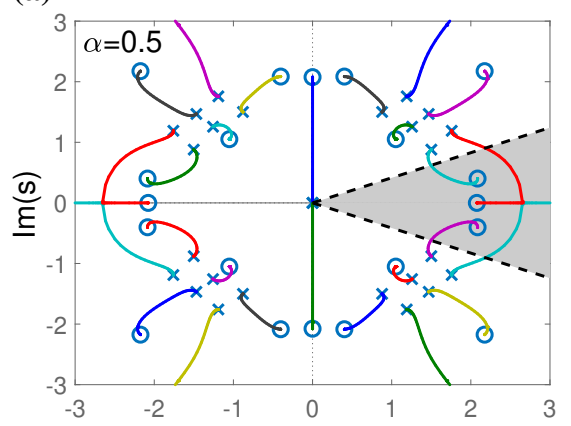

(b)

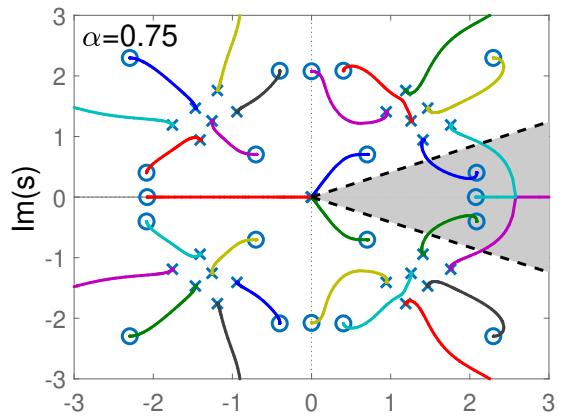

(c)

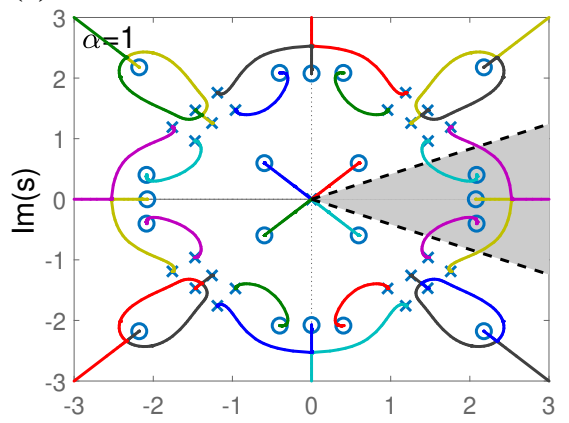

(d)

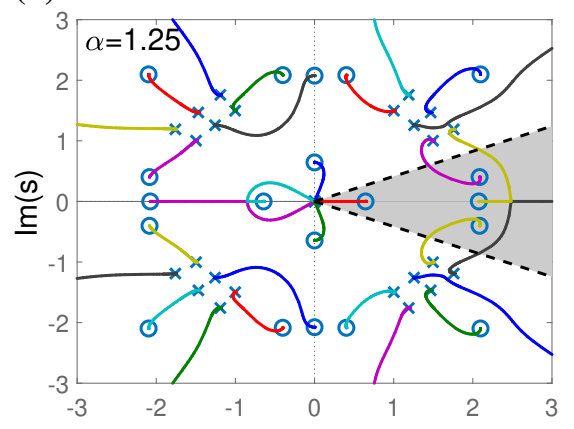

(e)

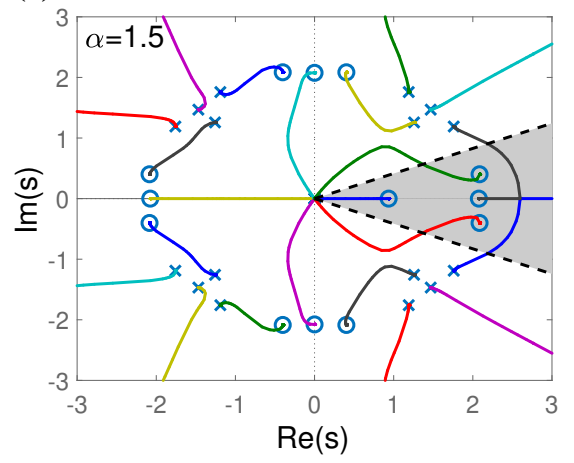

Figure 9. Root locus plots for the continuous model $H_{\alpha}^{c}(s)$ (10) controlling the plants $G_{\alpha}$ (2) with (a) $\alpha=0.5$, (b) $\alpha=0.75$, (c) $\alpha=1.0$, (d) $\alpha=1.25$ and (e) $\alpha=1.5$. A Padé approximant of the human effective delay was included in the transfer function. The colors are arbitrarily assigned to discriminate between the different branches for each pole. Some of the branches have a terminus at infinity. 


\section{Discussion}

Although the FIR model is proposed as a qualitative model of the human control response patterns, the control actions of the human and of the model were also compared. In Martínez-García and Gordon (2016) - and with the same data-set employed in this study - it was seen that on average humans produced a mean squared error of 0.155 with joystick control and 0.205 with steering wheel control.

For $G_{\alpha}(s)$ (2) with $\alpha \leq 1$ the relative mean squared error of the difference between human test subjects and model was of $0.0158(16 \%)$ and $0.0124(12 \%)$ for the joystick and the steering wheel respectively. These values are in agreement with the reported magnitude for the remnant in Roig (1962) $(\approx 10 \%)$

The evidence that the human subjects could still control in a stable manner the higher order fractional plants, while the linear controller cannot, suggests that a non-linear component or remnant is missing in this type of model. For classical linear transfer functions, the remnant or non-linear characteristics of a human-machine system are commonly regarded as negligible (McRuer \& Jex, 1967). Thus a possible explanation, for the instability of this model when $\alpha>1$, is that the remnant may be more significant for higher order fractional plants, as these are more difficult to control. Indeed some subjects manifested this opinion during the course of the experiments.

Although the linear equivalent system (5) is not enough for achieving stable control of higher order fractional systems ( 2 with $\alpha>1$ ), the linear part of the procedural memory pattern seems to have the same qualitative aspects as in the case when $\alpha \leq 1$ and stable control is achieved (Fig. 6). One possible explanation for this is that the human operator, besides pre-learned control patterns - which are generic for a wide category of plants, also introduces non-linear corrective actions to compensate for the deficiencies of those patterns, when they do not fully adjust to the controlled system.
While adding an additional term to the controller could stabilize the system, this would not tell much about the characteristics of human control, which was the main topic of this research. In general, modeling the remnant has not proven to be useful, due to the variability in human behavior (Jürgensohn, 2007).

Further, the proposed model only considers onedimensional inputs in a simple laboratory task. In realistic control tasks the visual scene will be more complex, and the relationship between control actions and visual memories would need to be categorized with respect to optical variables.

\section{Conclusions}

This paper was concerned with examining the role that visual memories play in producing adequate tracking control. A methodology was proposed to investigate procedural memory patterns found in human response while performing a manual control tracking task. The approach consists in fitting human response to a Finite Impulse Response (FIR) model, by means of a genetic algorithm. For this, we utilized data collected from ten human subjects controlling plants with five levels of different fractional order, and with a joystick or a steering wheel.

From the fitted parameters of the model, different conclusions have been drawn. Primarily, the human operator seems to rely on a characteristic memory pattern, which is dependent on the controlled plant. This pattern is consistent across different human subjects and different control devices. Also, it is consistent across different control modes: compensatory and pursuit. The pattern reflects how the human operator employs past observations of visually acquired perceptions, in order to predict the states of the plant. Moreover, it was shown how humans adapt the pattern when the order of the plant is increased: humans used a shorter memory span and higher gains for higher order plants. For the tested plants the pattern displays an average 
duration of $650 \mathrm{~ms}$ - a value that reflects the decay time and storage capacity of visual memories in a control task.

Further, the FIR model was reduced to a simplified lower order continuous time model. From the simplified model the stability of the whole human-machine system in closed-loop was assessed. It was shown that the fitted procedural memory pattern is unstable for plants with $\alpha>1$. However, for these plants the human subjects were able to achieve effective control with both control devices, albeit that with difficulty and lower performance. Hence, it is hypothesized that for higher order fractional plants the remnant characteristics that reflect non-linear behavior in human control are more significant; in the case of non-fractional and linear plants, the remnant effects are typically considered inconsequential.

These results are aimed to increase the repertoire of theoretical results on biofidelic human control, in particular in relation to human perception of optical variables. The applicability of these results is directed towards humanmachine interaction such as: realistic video game simulation or gaming performance evaluation; vehicle automation in highway driving; and developing testing technologies for vehicles in the field - that mimic adequately human driver behavior. One particular example is that of the so-called shared control systems, in which the control of a machine is shared between an intelligent system and a human operator. For these techniques to work effectively, the intelligent system needs to reproduce the overall characteristics of the human responses.

Future work will be directed to assess which optical variables humans use in realistic control tasks, such as driving a vehicle, and to implement biofidelic models that assist in shared control tasks. Further, the relation of the procedural memory patterns with event-related potentials in the frontal cortex is left as a potential expansion of this work, which would consist in obtaining electroencephalogram or functional near-infrared spectroscopy recordings from the motor cortex synchronous to the human control responses.
Another option is surface electromyography sensing in the biceps (Nacpil, Wang, Zheng, Kaizuka, \& Nakano, 2019).

\section{Key Points}

- Data recorded from human subjects controlling plants of different fractional orders are investigated.

- From the controlled actions elicited by humans, procedural memory patterns are identified and fitted to a Finite Impulse Response (FIR) model.

- The proposed FIR model is utilized as an analysis tool to estimate different properties of human manual control based on visual cues, such as the time scale of the procedural memory patterns.

- The stability of the whole human-machine system is assessed through fractional calculus theory.

\section{References}

Abbink, D. A., Carlson, T., Mulder, M., de Winter, J. C., Aminravan, F., Gibo, T. L., \& Boer, E. R. (2018). A topology of shared control systems - finding common ground in diversity. IEEE Transactions on HumanMachine Systems, 48(5), 509-525.

Anastasio, T. J. (1994). The fractional-order dynamics of brainstem vestibulo-oculomotor neurons. Biological Cybernetics, 72(1), 69-79.

Anderson, J. R., \& Lebiere, C. J. (2014). The atomic components of thought. Psychology Press, New York, USA.

Baker, J. E. (1987). Reducing bias and inefficiency in the selection algorithm. In Proceedings of the Second International Conference on Genetic Algorithms (Vol. 206, pp. 14-21).

Barfield, A., \& Dazzo, J. (1983). Multivariable control laws for the AFTI/F-16. In 22nd Aerospace Sciences Meeting (p. 237)

Beall, A. C., \& Loomis, J. M. (1996). Visual control of steering without course information. Perception, 
25(4), 481-494.

Bullier, J. (2001). Integrated model of visual processing. Brain Research Reviews, 36(2), 96-107.

Burdet, E., Osu, R., Franklin, D. W., Milner, T. E., \& Kawato, M. (2001). The central nervous system stabilizes unstable dynamics by learning optimal impedance. Nature, 414(6862), 446-449.

Burr, D. (1980). Motion smear. Nature, 284(5752), 164.

Card, S. K., Newell, A., \& Moran, T. P. (1983). The psychology of human-computer interaction. CRC Press, Boca Raton, FL, USA.

Chen, J. Y., \& Thropp, J. E. (2007). Review of low frame rate effects on human performance. IEEE Transactions on Systems, Man, and Cybernetics-Part A: Systems and Humans, 37(6), 1063-1076.

Clifford, C. W., \& Ibbotson, M. (2002). Fundamental mechanisms of visual motion detection: models, cells and functions. Progress in Neurobiology, 68(6), 409437.

Craik, K. J. (1947). Theory of the human operator in control systems (i). British Journal of Psychology. General Section, 38(2), 56-61.

Craik, K. J. (1948). Theory of the human operator in control systems (ii). British Journal of Psychology. General Section, 38(3), 142-148.

Donges, E. (1978). A two-level model of driver steering behavior. Human Factors: The Journal of the Human Factors and Ergonomics Society, 20(6), 691-707.

Gibson, J. J. (1950). The perception of the visual world. Houghton Mifflin, Oxford, UK.

Gregory, R. L. (1997). Eye and brain: The psychology of seeing. Princeton University Press, New Jersey, USA.

Griffiths, P. G., \& Gillespie, R. B. (2005). Sharing control between humans and automation using haptic interface: primary and secondary task performance benefits. Human Factors, 47(3), 574-590.

Hanneton, S., Berthoz, A., Droulez, J., \& Slotine, J.-J. E.
(1997). Does the brain use sliding variables for the control of movements? Biological Cybernetics, 77(6), 381-393.

Hollingworth, A., Richard, A. M., \& Luck, S. J. (2008). Understanding the function of visual short-term memory: transsaccadic memory, object correspondence, and gaze correction. Journal of Experimental Psychology: General, 137(1), 163.

Inga, J., Eitel, M., Flad, M., \& Hohmann, S. (2018). Evaluating human behavior in manual and shared control via inverse optimization. In Systems, Man, and Cybernetics (SMC), 2018 IEEE International Conference on (pp. 2699-2704).

Ito, K., \& Ito, M. (1975). Tracking behavior of human operators in preview control systems. Electrical Engineering in Japan, 95(1), 120-127.

Jagacinski, R. J., \& Flach, J. M. (2003). Control theory for humans: Quantitative approaches to modeling performance. CRC Press, Boca Raton, FL, USA.

Jürgensohn, T. (2007). Control theory models of the driver. In Modelling driver behaviour in automotive environments (pp. 277-292). Springer, London, UK.

Kase, S. E., Ritter, F. E., Bennett, J. M., Klein, L. C., \& Schoelles, M. (2017). Fitting a model to behavior reveals what changes cognitively when under stress and with caffeine. Biologically Inspired Cognitive Architectures, 22, 1-9.

Kawato, M. (1999). Internal models for motor control and trajectory planning. Current Opinion in Neurobiology, $9(6), 718-727$.

Kim, J. S., Greene, M. J., Zlateski, A., Lee, K., Richardson, M., Turaga, S. C., ... others (2014). Space-time wiring specificity supports direction selectivity in the retina. Nature, 509(7500), 331.

Magin, R. L. (2006). Fractional calculus in bioengineering. Begell House Redding, Danbury, USA.

Martínez-García, M., \& Gordon, T. (2016). Human 
control of systems with fractional order dynamics. In Systems, Man, and Cybernetics (SMC), 2016 IEEE International Conference on (pp. 2866-2871).

Martínez-García, M., \& Gordon, T. (2018). A new model of human steering using far-point error perception and multiplicative control. In 2018 IEEE International Conference on Systems, Man, and Cybernetics (SMC) (pp. 1245-1250).

Martínez-García, M., Gordon, T., \& Shu, L. (2017). Extended crossover model for human-control of fractional order plants. IEEE Access, 5, 27622-27635.

Martínez-García, M., Zhang, Y., \& Gordon, T. (2016). Modeling lane keeping by a hybrid open-closed-loop pulse control scheme. IEEE Transactions on Industrial Informatics, 12(6), 2256-2265.

Matignon, D. (1998). Generalized fractional differential and difference equations: stability properties and modelling issues. In Mathematical Theory of Networks and Systems Symposium (pp. 503-506).

McRuer, D. T., \& Jex, H. R. (1967). A review of quasilinear pilot models. Human Factors in Electronics, IEEE Transactions on, 8(3), 231-249.

Miall, R. C., Weir, D., \& Stein, J. (1993). Intermittency in human manual tracking tasks. Journal of Motor Behavior, 25(1), 53-63.

Nacpil, E. J. C., Wang, Z., Zheng, R., Kaizuka, T., \& Nakano, K. (2019). Design and evaluation of a surface electromyography-controlled steering assistance interface. Sensors, 19(6), 1308.

Pauwelussen, J. (2012). Dependencies of driver steering control parameters. Vehicle System Dynamics, 50(6), 939-959.

Pew, R. W. (2007). Some history of human performance modeling. In W. Gray (Ed.), Integrated models of cognitive systems (pp. 29-44). Oxford University Press, Oxford, UK.

Podlubny, I. (1998). Fractional differential equations: an introduction to fractional derivatives, fractional differential equations, to methods of their solution and some of their applications. Academic press, Cambridge, USA.

Ritter, F. E. (n.d.). Modeling human cognitive behavior for system design. In Digital human modelling and posturography (in prep.). Elsevier, Amsterdam, Netherlands.

Ritter, F. E., Baxter, G. D., Jones, G., \& Young, R. M. (2000). Supporting cognitive models as users. ACM Transactions on Computer-Human Interaction (TOCHI), 7(2), 141-173.

Ritter, F. E., Tehranchi, F., \& Oury, J. D. (2019). ACT-R: A cognitive architecture for modeling cognition. Wiley Interdisciplinary Reviews: Cognitive Science, 10(3), e1488.

Roig, R. W. (1962). A comparison between human operator and optimum linear controller rms-error performance. IRE Transactions on Human Factors in Electronics, $3(1), 18-21$.

Salvucci, D. D., \& Gray, R. (2004). A two-point visual control model of steering. Perception-London, 33(10), $1233-1248$.

Schmitt, F., Bieg, H.-J., Manstetten, D., Herman, M., \& Stiefelhagen, R. (2016). Exact maximum entropy inverse optimal control for modeling human attention switching and control. In Systems, Man, and Cybernetics (SMC), 2016 IEEE International Conference on (pp. 2807-2813).

Shinners, S. M. (1974). Modeling of human operator performance utilizing time series analysis. IEEE Transactions on Systems, Man, and Cybernetics, 4(5), $446-458$.

Squire, L. R. (2004). Memory systems of the brain: a brief history and current perspective. Neurobiology of Learning and Memory, 82(3), 171-177.

Tejado, I., Valério, D., Pires, P., \& Martins, J. (2013). 
Fractional order human arm dynamics with variability analyses. Mechatronics, 23(7), 805-812.

Tustin, A. (1947). The nature of the operator's response in manual control, and its implications for controller design. Electrical Engineers-Part IIA: Automatic Regulators and Servo Mechanisms, Journal of the Institution of, 94(2), 190-206.

Walter, W. G. (1964). Contingent negative variation: an electric sign of sensori-motor association and expectancy in the human brain. Nature, 230, 380-384.

Wang, Z., Zheng, R., Kaizuka, T., \& Nakano, K. (2018). Influence of haptic guidance on driving behaviour under degraded visual feedback conditions. IET Intelligent Transport Systems, 12(6), 454-462.

Wargo, M. J. (1967). Human operator response speed, frequency, and flexibility: A review and analysis. Human Factors, 9(3), 221-238.

Weiszer, M., Chen, J., \& Locatelli, G. (2015). An integrated optimisation approach to airport ground operations to foster sustainability in the aviation sector. Applied Energy, 157, 567-582.

Zgonnikov, A., \& Markkula, G. (2018). Evidence accumulation account of human operators' decisions in intermittent control during inverted pendulum balancing. In 2018 IEEE International Conference on Systems, Man, and Cybernetics (SMC) (pp. 716-721).

Zhang, Y., Martínez-García, M., \& Gordon, T. (2018).

Human response delay estimation and monitoring using gamma distribution analysis. In 2018 ieee international conference on systems, man, and cybernetics (smc) (pp. 807-812).

Miguel Martínez-García received a BSc degree in Mathematics from the Polytechnic University of Catalonia (UPC), Spain in 2013, a MSc in Advanced Mathematics and Mathematical Engineering (MAMME) from the same university in 2014, and a $\mathrm{PhD}$ degree in Engineering from the University of Lincoln, UK, in 2018 . He is currently a
Research Associate in Intelligent Systems at the Advanced Virtual Reality Research Centre (AVRRC), Loughborough University, UK. His research interests include Humanmachine Systems, Machine Learning, Artificial Intelligence and Applied Mathematics - such as Dynamical and Complex Systems.

Yu Zhang is currently a Senior Lecturer in Digital Engineering at the Department of Aeronautical and Automotive Engineering, Loughborough University, Loughborough, UK. She has obtained her BEng degree from the School of Aerospace Engineering and Applied Mechanics, Tongji University, Shanghai, China, and her MSc and $\mathrm{PhD}$ degrees from the Department of Civil Engineering, University of Nottingham, Nottingham, UK. Her recent research interests include Pattern Recognition, Condition Monitoring, Grey-box Modeling and the development of Data Analysis and Machine Learning algorithms, especially for industrial applications.

Tim Gordon received B.A. and M.A. degrees in mathematics from the University of Cambridge, U.K., in 1974 and 1975, respectively; in 1983 he was awarded a Ph.D. degree in applied mathematics from the Department of Applied Mathematics and Theoretical Physics at the same university. He is currently a Professor at the School of Engineering at the University of Lincoln, UK, and has held several senior academic posts including Professor of Mechanical Engineering at the University of Michigan, Ann Arbor, USA and Ford Professor of Automotive Engineering at the University of Loughborough, UK. 